\title{
Changing environmental conditions causes physical and molecular changes in tomatoes
}

Daniela D’Esposito

Francesca Ferriello

Alessandra Dal Molin

Gianfranco Diretto

Adriana Sacco

Andrea Minio

Amalia Barone

Rossella Di Monaco

Silvana Cavella

Luca Tardella

Giovanni Giuliano

Massimo Delledonne

Luigi Frusciante

Maria Raffaella Ercolano

Video Abstract

Keywords: Environment, Fruit quality, Metabolome, Network, Plasticity, Sensorial attributes, Solanum lycopersicum, Transcriptome

Posted Date: March 23rd, 2021

DOl: https://doi.org/10.21203/rs.3.rs-353575/v1

License: (9) This work is licensed under a Creative Commons Attribution 4.0 International License. Read Full License 


\section{Abstract}

Walking through the produce section at the grocery store, you are likely to find tomatoes of all shapes, sizes, and colors. One variety may be large and oblong with a hint of bitter flavor while another will have small, sweet fruits. This variance is primarily the result of genetic and chemical properties of the different varieties. But it turns out, environment also plays a role. A team of Italian scientists has shown that the molecular properties of tomatoes are strongly influenced by environmental factors such as temperature and moisture. And changing these factors can, in turn, have pronounced effects on the physical and culinary qualities of the fruits - an important finding considering the pace of current climate change. To tease apart the interaction between genetics, environment, and organoleptic traits, the research team grew three tomato varieties in two different locations. This exposed the plants to varying levels of moisture, soil acidity, and temperature, among other conditions. Environmental effects were gauged at three different levels: genetic, metabolic, and sensory. A panel of judges evaluated the tomatoes' sensory traits in terms of flavor, appearance, texture, and aroma. RNA sequencing and metabolomics analyses were then used to examine environmental effects at the genetic and chemical level. The investigators found that under certain environmental conditions, the plants responded with a cascade of genetic changes. While the three varieties varied in their genetic and metabolitic responses, things like appearance and taste were consistently affected. These findings highlight the complex interplay between genetics and the environment and provide insights into the mechanisms that regulate fruit quality variation. The implications of this work, however, go beyond pasta sauce and tomato soup. Understanding the genomic response of tomatoes to environmental stressors can help to better understand why some varieties are more resilient than others. In the face of ongoing climate change, that knowledge could give plant breeders insights into how best to develop this important food source. 